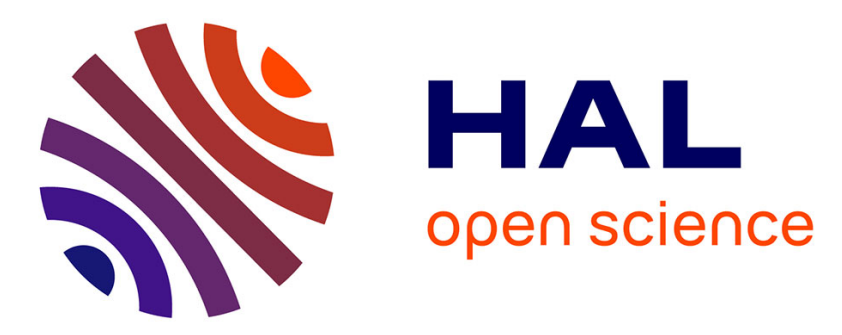

\title{
Inducing self-motion sensations in driving simulators using force-feedback and haptic motion
}

\author{
Guillaume Bouyer, Amine Chellali, Anatole Lécuyer
}

\section{To cite this version:}

Guillaume Bouyer, Amine Chellali, Anatole Lécuyer. Inducing self-motion sensations in driving simulators using force-feedback and haptic motion. VR 2017 - 19th IEEE Virtual Reality, Mar 2017, Los Angeles, United States. pp.84-90, 10.1109/VR.2017.7892234 . hal-01524537

\section{HAL Id: hal-01524537 \\ https://hal.science/hal-01524537}

Submitted on 23 May 2017

HAL is a multi-disciplinary open access archive for the deposit and dissemination of scientific research documents, whether they are published or not. The documents may come from teaching and research institutions in France or abroad, or from public or private research centers.
L'archive ouverte pluridisciplinaire HAL, est destinée au dépôt et à la diffusion de documents scientifiques de niveau recherche, publiés ou non, émanant des établissements d'enseignement et de recherche français ou étrangers, des laboratoires publics ou privés. 


\title{
Inducing Self-Motion Sensations in Driving Simulators Using Force-Feedback and Haptic Motion
}

\author{
Guillaume Bouyer* \\ IBISC, Univ. Evry Val d'Essonne, France
}

\author{
Amine Chellali ${ }^{\dagger}$ \\ IBISC, Univ. Evry Val d'Essonne, France
}

\author{
Anatole Lécuyer \\ Inria, France
}

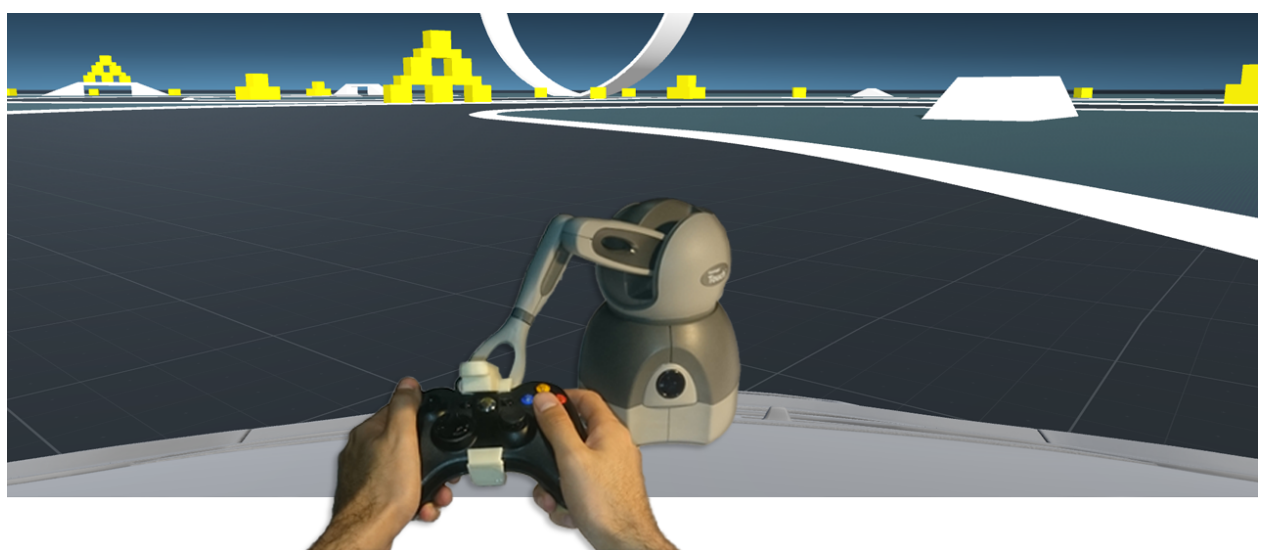

Figure 1: Our approach consists in simulating self-motion sensation during driving simulation with a force-feedback applied on the manipulated controller. Our prototype is focused on video game context: the user controls the navigation using a classical gamepad which is physically attached to the extremity of a standard force-feedback device. Haptic forces are proportional to the virtual acceleration of the vehicle in the simulation. As an example, braking and acceleration phases are simulated with longitudinal forces applied on the gamepad.

\begin{abstract}
Producing sensations of motion in driving simulators often requires using cumbersome and expensive motion platforms. In this article we present a novel and alternative approach for producing self-motion sensations in driving simulations by relying on hapticfeedback. The method consists in applying a force-feedback proportional to the acceleration of the virtual vehicle directly to the hands of the driver, by means of a haptic device attached to the manipulated controller (or a steering wheel). We designed a proofof-concept based on a standard gamepad physically attached at the extremity of a standard 3DOF haptic display. Haptic effects were designed to match notably the acceleration/braking (longitudinal forces) and left/right turns (lateral forces) of the virtual vehicle. A preliminary study conducted with 23 participants, engaged in gamepad-based active VR navigations in a straight line, showed that haptic motion effects globally improved the involvement and realism of motion sensation for participants with prior experience with haptic devices. Taken together, our results suggest that our approach could be further tested and used in driving simulators in entertainment and/or professional contexts.
\end{abstract}

Keywords: Driving Simulation, Self-motion, Haptic, Forcefeedback.

Index Terms: H.1.2 [User/Machine Systems]: Human factors; H.5 [Information Interfaces and Presentation]: Artificial, augmented, and virtual realities-Evaluation/methodology; H.5.2

\footnotetext{
*e-mail: guillaume.bouyer@ibisc.univ-evry.fr

†e-mail: amine.chellali@ibisc.univ-evry.fr

‡e-mail: anatole.lecuyer@inria.fr
}

[User Interfaces]: User-centered design-Haptic I/O

\section{INTRODUCTION}

Driving simulators are an important and early application of virtual reality technologies $[2,16]$. As for today, driving simulators are extensively used in entertainment (e.g., video games, arcade, theme parks), but also professional contexts. They can be used for studying the behaviour of the driver and/or the vehicle $[6,11,13]$, for designing driving aid systems [14], for designing and testing accessibility of embedded human-computer interfaces $[25,26]$ or road infrastructures [28].

In order to increase the realism of the driving simulation and to provide realistic sensations of self-motion, these simulators can rely on motion platforms [23], which are often bulky and expensive. Alternative solutions have been occasionally explored in the literature such as using vibratory feedback [24,27] or galvanic vestibular stimulations $[1,17]$. However, these techniques often fail at reproducing realistic self-motion sensations in a comfortable and sustainable manner.

Interestingly, in the context of multimedia and audiovisual experiences, some researchers have recently shown that using forcefeedback applied to the hands of a seated user could succeed in generating strong sensations of self-motion during a passive video viewing [7-9, 20]. This phenomenon, called "haptic motion", was demonstrated to be successful at producing a higher sensation of vection (illusion of self-motion) during virtual 3D navigations compared to the use of visual-feedback alone [20]. However, these results were obtained in a passive context, i.e., the user was passively perceiving the sensory feedbacks and was not able to control the virtual navigation - which is mandatory in a driving simulation.

In this paper, we propose a novel approach for driving simulators which provides sensations of self-motion by relying on forcefeedback (Figure 1), at low-cost, compared to the traditional motion 
platforms. It extends the "haptic motion" paradigm to the context of driving simulation, and to the situation of an "active" navigation. We have designed a proof-of-concept system focusing on the video game context. Our prototype is based on a gamepad fixed at the extremity of a standard 3DOF haptic device. Haptic effects were designed to simulate notably the acceleration/braking of the virtual vehicle with longitudinal forces, and the left/right turns with lateral forces. We have also conducted a pilot study to assess the subjective experience of 23 participants when using our system and being exposed to haptic motion effects.

In the remainder of this paper we first present related work on self-motion simulation. Then we introduce the concept and general ideas of our approach, followed by the description of the components of our proof-of-concept system, and the model for forcefeedback calculation. Then, we present the design and the results of our preliminary study conducted with naive participants. The paper ends with a discussion and a general conclusion.

\section{Related Work}

The sensation of self-motion is essential for driving simulation but also for numerous VR applications (games, training, virtual visits, urban projects reviews, etc.) [18]. The self-motion sensation comes from the combination of cues from various sensory channels: visual, vestibular, tactile, proprioceptive, audio, etc. Using visual feedback remains a simple way to induce a sensation of movement. It can generate vection, a well-known illusion of self-motion [3]. Vision was also found to be well adapted to detect velocity but not acceleration [21].

The most commonly used solution for creating self-motion sensations in driving simulators is vestibular or motion platforms [30]. The most advanced systems feature a driving cabin including all or part of an instrumented vehicle and a 360 degrees visual display, all mounted on a large robotic platform. The use of physical control and display devices such as steering wheels, pedals, dashboards, and mirrors are expected to enhance the fidelity of the users driving experience. These physical platforms are complemented by realistic vestibular feedback to give the illusion of longitudinal and lateral accelerations. However, these platforms come with several drawbacks. The first one is related to their size. Indeed, in order to generate vestibular feedback for large-scale displacements, these platforms require a large workspace (tens of square meters). The other main issues include: their cost (hundreds or sometimes millions of USD [4]), and the complexity of their control system [10].

Alternative solutions for producing self-motion sensations have been explored, based on various sensory stimulations $[1,5,12,17$, $19,25,27]$. This includes vibrotactile interfaces, proprioceptive interfaces (e.g., treadmill) or galvanic stimulation that electrically stimulates the vestibular system via the inner ear. Nevertheless, some issues can occur such as: discomfort for long-term use, a constrained direction of stimulation, or the lack of information on the magnitude of the perceived acceleration.

In a context of passive virtual navigation, Ouarti et al. [20] have recently shown that a haptic force-feedback in the hands can dramatically improve the whole-body sensation of motion when compared with a visual feedback of displacement alone. The authors called this effect "haptic motion". More particularly, hapticfeedback had a significant effect on the frequency of occurrences, the duration, the onset of illusion, and also on the subjective intensity of the sensation of self-motion. Different models for force calculation were compared. A haptic-feedback proportional in magnitude to the 3D acceleration of the virtual camera and in the same direction, combined with visual feedback, was found to have the most important impact on self-motion perception. A force-feedback proportional to the velocity of the virtual camera had a smaller effect on self-motion sensation but still more influence than no hapticfeedback at all. Interestingly enough, the researchers also compared force-feedbacks displayed in the same versus opposite direction as the visual motion, and they found that both models were equally selected by participants $(50-50 \%)$.

In the context of multimedia applications, Danieau et al. [7-9] have proposed an application of "haptic motion" to increase the sensation of motion during a passive video viewing experience. They designed the "HapSeat" system (a haptic seat), which applies forcefeedback at the level of the armrests and headrest (on users hands and head) in synchronization with the video or camera movements. The presence of this force-feedback was found to significantly enhance the quality of the user experience, more particularly the realism, the immersion and the users satisfaction. But these results were obtained in a passive (video) viewing experience, and not for an active context, such as with driving simulators, in which the user can control the navigation.

Regarding consumer applications, several motion platforms emerge for driving videogames (e.g. Accelid DS $3^{1}$ ). But they are still cumbersome and relatively expensive. Force-feedback controllers have also been specifically designed for the videogame market (e.g. 1DOF haptic steering wheel for racing games, 2 or 3 DOF Joysticks for flying simulations or Novint Falcon). However, haptic-feedback is not used here to reproduce movements and self-motion sensations. It is mainly used to increase the realism of the manipulation of the simulated controller. For example, current force-feedback steering wheels used in videogames and/or driving simulators are generally actuated around the roll axis only, and simulate the sensations related to the manipulation of a real steering wheel. Force-feedback can also be used to transmit timely information or simulate special effects and specific events related to the interactions of the vehicle with the environment (collisions, sliding, skidding, or road departures). To the authors best knowledge, none of these devices have already been used to improve the sensation of self-motion.

Therefore, in this context, our objective is to leverage the "haptic motion" approach in order to improve the sensation of self-motion in the situation of an active driving simulation.

\section{A Novel Approach for Inducing Self-motion Sensation in Driving Simulators With Force- FEEDBACK}

\subsection{Concept and General Ideas}

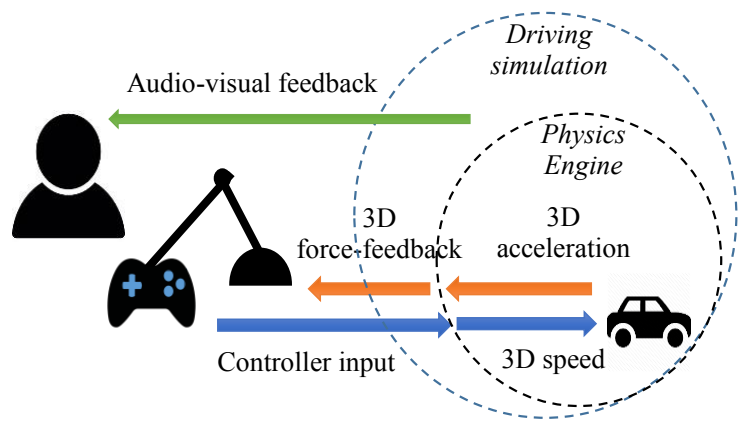

Figure 2: Our general architecture for inducing self-motion sensations in driving simulations using force-feedback.

When we are seated behind the steering wheel of a moving car, our body perceives several forces and motion information. Our vestibular sense perceives: (i) the forces related to longitudinal acceleration during acceleration and braking phases, (ii) the forces related to the lateral acceleration during turns, and also (iii) the

\footnotetext{
${ }^{1}$ http://www.accelid.co.at/
} 
forces related to gravity. Second, our proprioceptive system perceives the forces related to the contacts with the car components (steering wheel, pedals, seat, etc.).

In order to increase the sensations of self-motion in driving simulators, we propose an approach which preserves proprioceptive sensations and transmits several vestibular effects via the users hands through the haptic channel.

Our general concept consists in applying a force-feedback in the hands of the user, via the manipulated controller - steering wheel, gamepad, joystick, etc. This force-feedback cue is related to the behaviour motion of the virtual vehicle, and is expected to induce a strong sensation of self-motion. Such kind of effect, called "haptic motion" [20], was found to be very successful in a passive context, i.e., the user experiencing a predefined navigation on which he/she has no control. In our case, in the context of driving simulation, the user commands the navigation of the virtual vehicle. A main advantage of this approach is that it allows to generate accelerations that can be temporally longer or infinite without being limited by the joint stops of the interface. The user can resist to the forcefeedback of a constant acceleration, without exceeding the device workspace [20]. In addition, this approach can theoretically cover all spatial orientations with a 6DOF haptic-feedback (longitudinal, lateral, vertical accelerations), which is complex to generate with a vestibular stimulation platform.

The main components and architecture of our approach are depicted in Figure 2. Similar to a car or a conventional driving simulator, the user controls the virtual vehicle by means of a controller (here a gamepad, but possibly any input device including steering wheels and pedals). Using this input, the VR driving simulation computes the position and acceleration of the virtual vehicle based on its physical parameters and the characteristics of the $3 \mathrm{D}$ environment (slope, collision, gravity, friction, etc.). A force model transforms the 3D acceleration of the virtual vehicle into a 3D force which is fed sent back to the user by means of a force-feedback device attached to the controller. The controller and the haptic device can also be engineered in an integrated manner within an all-in-one hardware system (e.g. a steering wheel with longitudinal actuators). The number of degrees of freedom of the force-feedback device somehow constrains the variety of haptic motion effects that can be simulated. A $1 \mathrm{DOF}$ force-feedback can apply longitudinal forces and to simulate acceleration and braking phases, whereas a 3DOF device simulates additionally the turns and the vertical motions.

Besides, as in any driving simulator, the user experience can be augmented by using other sensory feedbacks, such as auditory effects that can notably increase the perception of accelerations (engine noises) and brakings (screeching tires).

\subsection{Proof-of-concept}

We have designed a proof-of-concept of our approach which is shown in Figure 3. The force-feedback is based on a 3DOF haptic device (Geomagic Touch (available for about $\$ 1800$ USD). The input device is a gamepad (Microsoft XBox 360). It is mounted on the haptic device using a custom 3D printed holder and a jack connector (see Figure 4). The control techniques of the virtual navigation are based on classical gamepad mappings (see Figure 5.a). The acceleration/braking of the virtual vehicle are controlled using the gamepad right/left analog triggers (moving the virtual vehicle forward/backward in the driving simulation). The steering (left/right) is controlled with the left thumbstick.

The virtual environment is displayed on a monitor (37 inches) with integrated speakers. The virtual reality application was designed using Unity 3D with C\#. The simulation is based on a driving game freely available in the Unity 3D standard assets ${ }^{2}$. The force-feedback was designed in Unity 3D using Kirurobos C\#

${ }^{2}$ www.assetstore.unity3d.com/en/!/content/32351

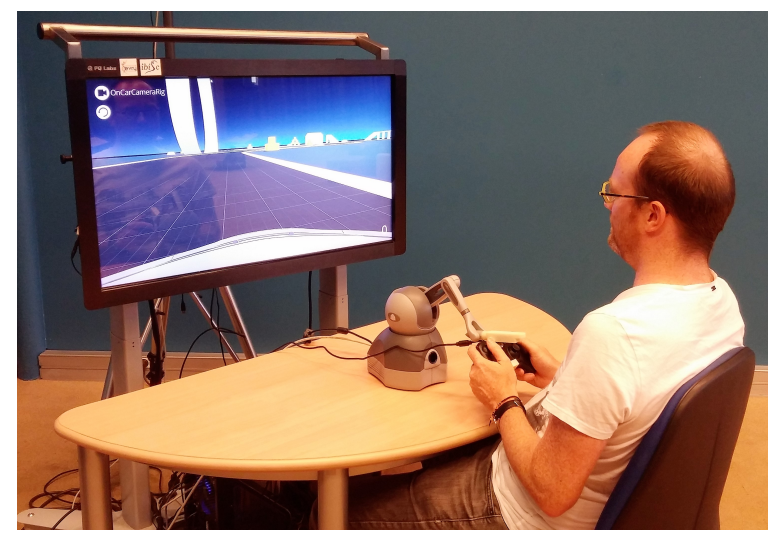

Figure 3: Proof-of-concept setup, based on a large screen and a gamepad attached to a 3DOF haptic device.
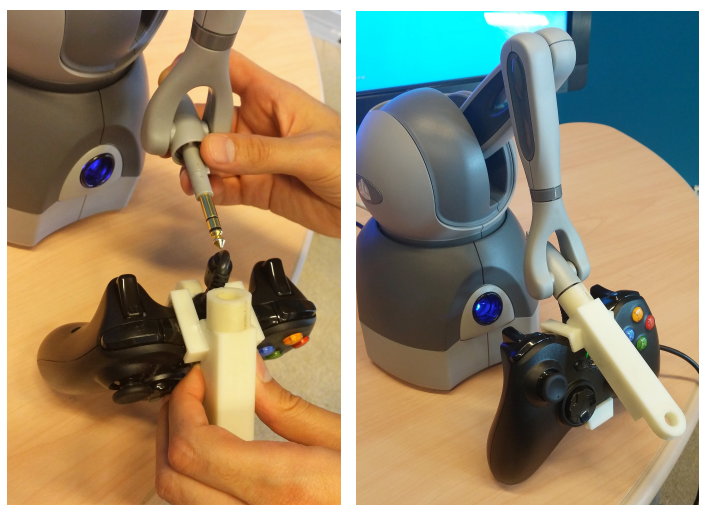

Figure 4: Assembly of the gamepad on the 3DOF haptic device with custom 3D printed holder and jack connector.

wrapper for the Geomagic Touch haptic Device [15]. The computation of forces relies on the Unity 3D integrated physics engine.

\subsection{Force-feedback Model}

The force-feedback model describes how the commands of the user are exploited to compute the "motion force-feedback" (see Figure 5). The user controls the speed and the direction of the virtual vehicle via the controller (see Figure 5.a). Based on these inputs, as well as the physical characteristics of the vehicle, and the virtual environment, the simulation calculates and updates the position/orientation of the vehicle. The resulting $3 \mathrm{D}$ velocity and $3 \mathrm{D}$ acceleration are then computed in the vehicles frame of reference (see Figure 5.b). Each XYZ component of the acceleration is then filtered using a low pass filter, scaled, and transmitted to the haptic device according to its own frame of reference (see Figure 5.c).

The straightforward computation of the final haptic force is given in Equation 1, with $\vec{F}$ the force-feedback output, $\vec{a}$ the acceleration of the virtual vehicle and $\vec{K}$ the gain vector. This allows us to set a different gain factor for each axis, which can be notably used to enhance longitudinal effects compared to the lateral ones, or to remove gravity effects.

$$
\vec{F}=\vec{K} \cdot \vec{a}
$$

We propose two distinct force-feedback models depending on the direction of the force compared to the direction of the 3D acceleration of the vehicle: "same direction" model (HSD) and "opposite direction" model (HOD). Indeed, it was previously observed in 


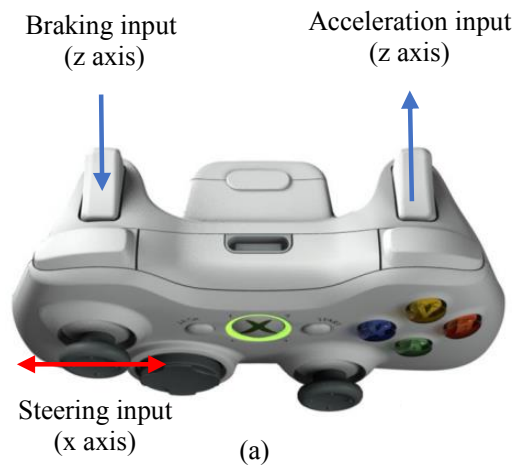

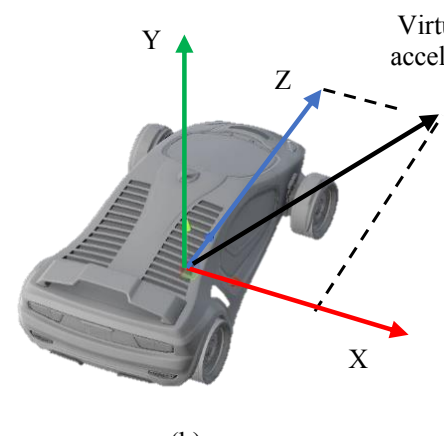

(b)

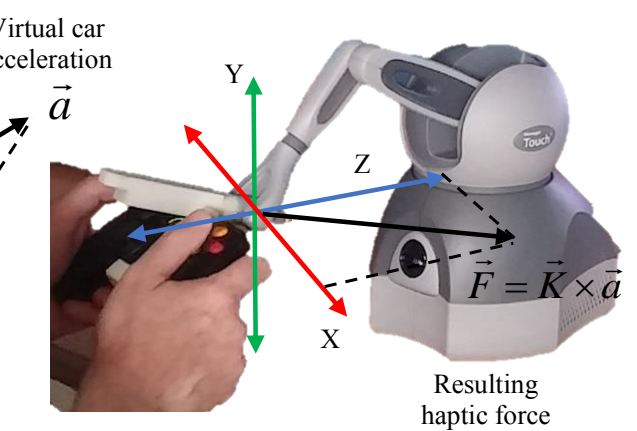

(c)

Figure 5: Close-up on the different steps of our force-feedback mode reference frame. (c) Haptic force-feedback device reference frame.

(a) Classical car control mapping on the gamepad. (b) Virtual vehicle

a passive context that the preferred direction of haptic motion effect seems randomly distributed among participants [20].

We can notice interesting connections between real driving and our haptic approach (see Figure 6). When holding the steering wheel in a car that accelerates, the body experiences a backward force, which stretches out the arms, pushing him away from the steering wheel. Similarly, when displayed in the same direction as the virtual acceleration, our haptic-feedback pulls the hands forward, which stretches out the users arms. Conversely, when slowing down or braking, the body is pushed closer to the steering wheel, and our haptic effect pushes the hands towards the body. During turns, the body undergoes the centrifugal forces that tend to move it towards the outside of the turn, which shifts it in relation to the hands. The HSD model moves the users hands towards the inside of the turn. In this case, the force-feedback mimics the car displacement and the body-hand geometric relationship is preserved. On the contrary, when forces are displayed in the opposite direction (HOD) as the acceleration, we transfer on the hands the forces normally applied on the body.

Interestingly, our approach can be used to convey other motion sensations than longitudinal and lateral displacements. Indeed, as the model directly transmits the 3D acceleration of the virtual vehicle, the user can naturally (consequently) perceive: collisions, gravity effects (e.g. ramp jump, vertical drops), or any other motion caused by the virtual environment (such as bumps).

\subsection{Results}

Our proof-of-concept works with no measurable impact on the original framerate ( 75FPS) of our driving simulation application and we did not observe instability issues concerning haptic rendering (on a standard PC with a gaming graphics card). Figure 7 illustrates the resulting forces computed and sent back to the user in two different situations: (a) a linear acceleration/braking phase, and (b) a series of left/right turns. In our driving simulation, the speed model of the physics engine is nonlinear, and the full braking is more powerful than the full acceleration (the force is instantly reversed when braking). One can observe the potentially long duration of the force application (15s of acceleration in this example) although the size of the haptic workspace is smaller than $150 \mathrm{~mm}$. Actually, we found that the user naturally tends to resist to the force-feedback in order to keep control over the vehicle. This confirms one advantage of our approach compare to vestibular platforms: the possibility to exert stimulations for a long period of time. Figure 7.b shows the lateral components of the virtual velocity and force, on a sample sequence of turning left then right at a constant longitudinal velocity. It illustrates the sudden changes of the lateral speed during turns which result in acceleration peaks.

As side remarks, we could note that frontal collisions can lead to

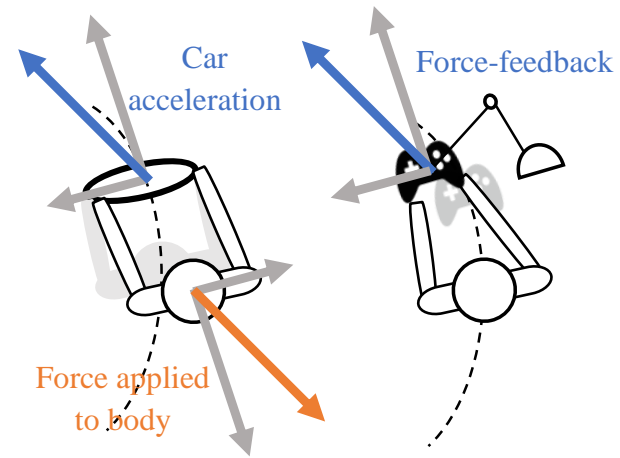

(a) Real Driving

(b) Same Direction experience. Haptic model (HSD)

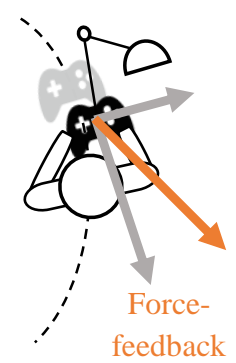

(c) Opposite

Direction Haptic model (HOD)
Figure 6: Illustration of two force-feedback models. With Same Direction Haptic model (b), force-feedback has the same direction as car acceleration and mimics the car displacement. With Opposite Direction Haptic model (c), force-feedback is in opposite direction and mimics the body displacement.

instantaneous drops in speed which are thus not always transmitted to the haptic device. But other motions due to bumps or ground textures generate reliable forces and strong sensations along the vertical axis.

\section{USER STUdY}

We conducted a user study to assess the influence of haptic effects on the user experience during a simple driving simulation: a straight road with a sequence of acceleration/braking phases. We used a questionnaire to compare the user experience in different conditions: in presence of haptic-feedback (HSD or HOD models) or not (control condition).

\subsection{Participants}

A total of 23 naive participants ( 18 male, 5 female; 22 right-handed, aged 22-47, mean=27.7, SD=7.5) participated in this study. They were all students or staff from a university. Eleven of them reported a previous experience with haptic devices while the twelve others never used any haptic device before. Nine reported playing video games frequently (at least once a week). None of them have reported a previous experience with motion and driving simulators. 


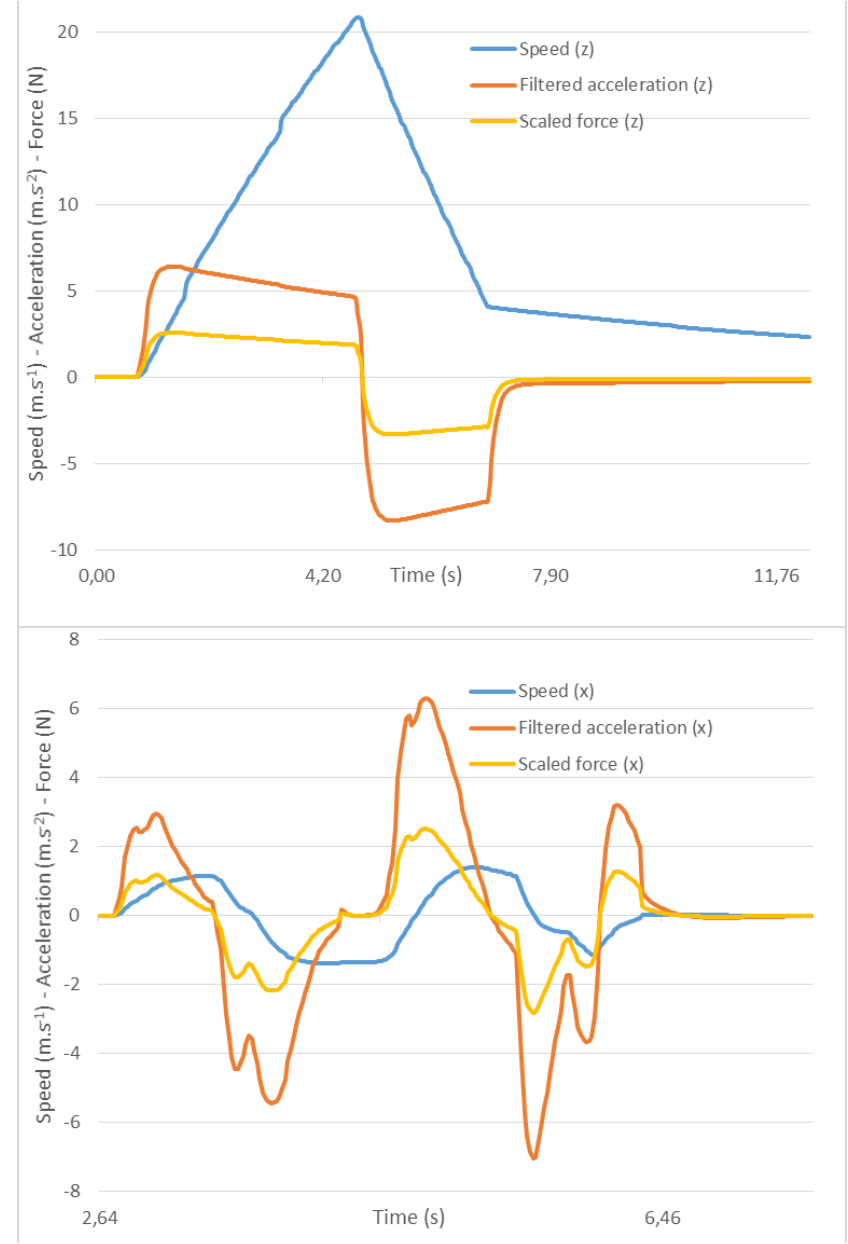

Figure 7: Examples of virtual speeds, accelerations and forces sent back to the user during (top) a sequence of acceleration and braking and (bottom) a sequence of left and right turns (with haptic gain $K=$ 0.4 and low-pass filter $R C=0.1$ ).

\subsection{Experimental Design}

The experiment used a within-subject design with one independent variable - the haptic-feedback model with three conditions: no haptic-feedback (Control Condition, CC), Same Direction Haptic model (HSD) with forces displayed in the same direction as those of the virtual car displacement, Opposite Direction Haptic model (HOD) with forces displayed in the opposite direction as those of the virtual car displacement. The haptic gain $K$ in both conditions is equal to 1 .

\subsection{Task and Procedure}

Prior to performing the experiment, the participants completed a questionnaire detailing the demographics. Then, they were asked to read the experimental instructions sheet. After that, they were seated in front of the monitor and were asked to hold the gamepad in a comfortable manner. They were then allowed to freely drive a car within a virtual circuit to become familiar with the system. During this familiarization period, all the force-feedback conditions were displayed during 30s. After that, the actual experiment started.

The participants were asked to drive a car in a simplified virtual circuit with only a straight line. At the beginning of each trial, a first message was displayed on the screen asking the participants to accelerate. Once the virtual car reached a predetermined position,
Table 1: Selected items for Witmer and Singer presence questionnaire [29].

\begin{tabular}{|l|l|}
\hline Code & Question \\
\hline Q1 & $\begin{array}{l}\text { How realistic was your sense of movement inside the } \\
\text { virtual environment? }\end{array}$ \\
\hline Q2 & $\begin{array}{l}\text { How much did your experiences in the virtual environ- } \\
\text { ment seem consistent with your real world experiences? }\end{array}$ \\
\hline Q3 & $\begin{array}{l}\text { How involved were you in the virtual environment ex- } \\
\text { perience? }\end{array}$ \\
\hline Q4 & $\begin{array}{l}\text { How natural did your interactions with the environment } \\
\text { seem? }\end{array}$ \\
\hline Q5 & $\begin{array}{l}\text { How much delay did you experience between your ac- } \\
\text { tions and expected outcomes? }\end{array}$ \\
\hline Q6 & $\begin{array}{l}\text { How much did the control devices interfere with the } \\
\text { performance of assigned tasks? }\end{array}$ \\
\hline
\end{tabular}

a second message was displayed to ask the participants to brake. After the car reached another predetermined position, the first message was displayed again to ask them to accelerate, and so on. A total of 3 acceleration and 3 breaking phases were performed for each trial. A total of 3 trials were performed per condition. After performing the 3 trials for one condition, participants were asked to fill in a questionnaire to evaluate their user experience for this specific condition. After that, they were asked to perform the same task for the next condition. After performing all the trials for all the 3 conditions, participants were asked to fill in a final questionnaire to compare the 3 experimental conditions and to give their feedback on the experiment. To avoid any learning effect, the presentation order of the 3 conditions was counterbalanced.

\subsection{Data Collection and Analysis}

We have selected a set of relevant items from the Witmer and Singer presence questionnaire [29] to evaluate the users subjective experience (Table 1). A 7-point Likert scale was used for each question (Completely disagree/not satisfactory to completely agree/very satisfactory). The participants' answers were grouped and a mean rating score was calculated for each item. To compare the mean scores for each condition, the Friedman and Wilcoxon tests were used (Non-parametric tests for ordinal data). In addition, participants were asked to rank the three haptic conditions according to their preference. Finally, the participants were given the opportunity to comment on their experience with the system.

\subsection{Results}

Answers of participants to the 6 selected questions are summarized on Table 2. The Friedman tests show a significant effect of the haptic-feedback on the realism of movements and on the involvement in the virtual world experience. No other significant differences were observed. The pairwise comparisons using Wilcoxons tests show that participants felt the movements more realistic in presence of haptic-feedback (both HSD and HOD conditions) than without ( $C C$ condition) $(\mathrm{Z}=-2.30, \mathrm{p}=0.02 ; \mathrm{Z}=-2.02, \mathrm{p}=0.04)$. Moreover, the tests show that participants felt more involved in the virtual world experience in the HSD and HOD conditions than in the $\mathrm{CC}$ condition $(\mathrm{Z}=-2.16, \mathrm{p}=0.03 ; \mathrm{Z}=-2.83, \mathrm{p}=0.005)$. No significant differences were found between the two haptic conditions.

We have compared participants answers according to their frequency of playing video games and their experience with haptic devices (Figure 8). Results show no significant effect of the frequency of playing video games while a significant effect of haptic-feedback on the realism of movements (Q1) was found only for participants who had a previous experience with haptic devices. Moreover, a 
Table 2: Questionnaire results.

\begin{tabular}{|l|l|l|l|l|l|}
\hline Questions & \multicolumn{3}{|c|}{ Mean scores } & \multicolumn{2}{l|}{ Friedman test } \\
\hline & CC & HSD & HOD & $\chi$ & $\begin{array}{l}\text { P- } \\
\text { Values }\end{array}$ \\
\hline $\begin{array}{l}\text { Q1 - Realism of } \\
\text { movement }\end{array}$ & $\begin{array}{l}3.63 \\
(1.42)\end{array}$ & $\begin{array}{l}4.48 \\
(1.46)\end{array}$ & $\begin{array}{l}4.41 \\
(1.15)\end{array}$ & 6.24 & 0.04 \\
\hline $\begin{array}{l}\text { Q2 - Consistence } \\
\text { with real world }\end{array}$ & $\begin{array}{l}3.65 \\
(1.77)\end{array}$ & $\begin{array}{l}4.22 \\
(1.59)\end{array}$ & $\begin{array}{l}4.26 \\
(1.48)\end{array}$ & 3.51 & 0.17 \\
\hline $\begin{array}{l}\text { Q3 - Involvement } \\
\text { in VE experience }\end{array}$ & $\begin{array}{l}4.17 \\
(1.70)\end{array}$ & $\begin{array}{l}5.00 \\
(1.51)\end{array}$ & $\begin{array}{l}5.13 \\
(1.58)\end{array}$ & 7.37 & 0.02 \\
\hline $\begin{array}{l}\text { Q4 - Naturalness } \\
\text { of interaction }\end{array}$ & $\begin{array}{l}4.52 \\
(1.47)\end{array}$ & $\begin{array}{l}4.74 \\
(1.29)\end{array}$ & $\begin{array}{l}4.52 \\
(1.47)\end{array}$ & 0.91 & 0.63 \\
\hline $\begin{array}{l}\text { Q5 - Perception of } \\
\text { delay }\end{array}$ & $\begin{array}{l}2.52 \\
(1.56)\end{array}$ & $\begin{array}{l}2.52 \\
(1.56)\end{array}$ & $\begin{array}{l}2.30 \\
(1.43)\end{array}$ & 0.80 & 0.66 \\
\hline $\begin{array}{l}\text { Q6 - Interference } \\
\text { of control device }\end{array}$ & $\begin{array}{l}3.65 \\
(1.61)\end{array}$ & $\begin{array}{l}3.48 \\
(1.86)\end{array}$ & $\begin{array}{l}3.78 \\
(1.93)\end{array}$ & 0.03 & 0.98 \\
\hline
\end{tabular}

significant effect of haptic-feedback on the involvement in the virtual world experience (Q3) was found only for participants with no previous experience with haptic devices. Pairwise comparisons using Wilcoxons tests show that participants who had previously used haptic devices felt the movements more realistic in the HSD condition than in the $\mathrm{CC}$ condition $(\mathrm{Z}=-2.23, \mathrm{p}=0.02)$. On the other hand, participants who had never used haptic devices felt more involved in the virtual experience in the HSD and HOD conditions than in the $\mathrm{CC}$ condition $(\mathrm{Z}=-2.05, \mathrm{p}=0.04 ; \mathrm{Z}=-2.52, \mathrm{p}=0.01)$. No other significant differences were found.

Finally, the results indicate that $86 \%$ of participants preferred the haptic-feedback conditions (HSD or HOD) and only $13 \%$ preferred the condition without any haptic-feedback (CC). The experience with haptic devices and video games had no significant effect on these choices. Regarding the distribution accross haptic models, our results show that both models were equally appreciated, with $43 \%$ of participants preferring each of them.

\section{Discussion}

The results of our user study suggest that our approach could succeed in improving the user experience during a simple driving simulation (straight line). A great majority of our participants (86\%) have preferred performing the driving task with haptic-feedback regardless the haptic model used. Besides, both haptic models significantly increased the perceived involvement in the virtual world experience and the perceived realism of movement compared to the no haptic-feedback condition. We also received very positive comments from participants after the experiment: "It was amazing", "I lived this experience as if it was real", "When I was accelerating, I had the sensation of being glued to the seat as in a real car".

Our observations during the experiment show that since the user is active, he/she holds quite strongly the controller to be able to press the buttons. Therefore, he/she naturally resists to the forces. Moreover, the perceived naturalness of interaction, the interferences of control device, and the perception of delays between inputs and outputs were not significantly influenced by the presence of haptic effects (questions Q4, Q5, and Q6 with similar scores). This can suggest that adding our haptic-feedback does not have a negative effect on interaction during the driving experience. Informal tests conducted during turn sequences showed similar results, but dedicated experiments are needed to confirm these findings.

Our results indicate also that the haptic effects were not perceived the same way by all participants. Indeed, the effects increased the involvement of participants who had never used hap-

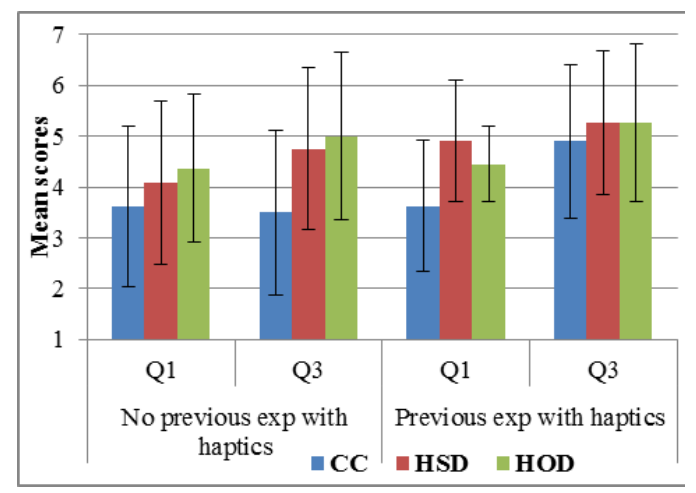

Figure 8: Answers of participants according to their previous experience with haptic devices (error bars represents the standard deviations).

tic devices, whereas they increased the realism of movements for those who had previously used them (but only for the HSD condition). This could be explained through the Rasmussens Skill, Rule and Knowledge model of human behaviour [22]. This model claims that the degree of conscious control exercised by the individual over his/her activities, depends on the degree of familiarity with the task and the environment. According to this model, the Knowledgebased level of behaviour requires the highest degrees of conscious involvement and attention and is observed when a person faces a new situation (e.g. a trainee at the beginning of his/her training). This could have been the case for our participants with no experience with haptics. This led them to focus more on this novel aspect of the situation (haptic interaction) and thus to be more involved in the virtual environment experience. On the other hand, the skillbased level of behaviour requires the lowest level of conscious involvement and is characterized by highly routinized and automated activities. This is the case of experts performing a task in a familiar environment. This could have been the case of our participants with a previous experience with haptics. They were less surprised by haptic interactions and were able to focus more on the remaining aspects of the situation, such as the perception of movement. However, the realism of movement was increased when the forces are displayed in the same direction as acceleration only for users with a previous experience with haptics. Thus, more in depth analyses are needed to better understand this phenomenon.

Our results showed no significant differences in terms of preference between the two haptic models. Half of our participants preferred when forces are in the same direction as acceleration, and the other half preferred the opposite. This result confirms observations made in previous studies on "haptic motion" $[8,20]$ but, in our case, for an "active" navigation. Our observations show that some participants found that the displayed haptic-feedback was sometimes "reversed" depending on the model. For instance, they were expected to be pushed backward instead of being pulled forward when the virtual vehicle was moving forward. One participant even commented after HOD condition that the acceleration sensation was really interesting and was similar to what one can feel during real world driving. Braking seems a little strange, though" while he commented after the HSD condition that "the acceleration sensation was almost disturbing and not necessarily similar to the driving experience. However, braking is more similar to what one can experience in real world". Thus, this participant always preferred when forces pushed hands back to the body. Future work seems necessary to further characterize this variability and the potential influence of users profile. Meanwhile, user should be left with the possibility to choose the direction of haptic effects (similarly to the selection of mouse controls in a first-person shooter game). 
As for system improvements, our participants suggested that "haptic forces should be applied also on the chair". Another participant thought that the simulation lacks "gear shifting sensations". Finally, a third participant thought that "having a steering wheel and pedals to control the vehicle will improve the realism of the driving experience as compared with the gamepad". In addition to these improvements, we believe that, for future work, complementary user studies could be conducted. We could first assess our approach in other driving simulation contexts (e.g. during turns, or in complex trajectories) taking into account the driving performance, for instance. It could also be interesting to compare our approach with gaming motion platforms and/or steering wheels.

\section{Conclusion}

We have proposed an approach that leverages the "haptic motion" paradigm for producing self-motion sensations in driving simulators, where the user actively controls the displacement of a vehicle. Our method consists in applying a force-feedback proportional to the acceleration of the virtual vehicle directly in the hands of the driver, by means of a haptic device attached to the manipulated controller. It permits generating long and multi-directional self-motion stimulations. Haptic effects were designed to match notably the acceleration/braking (longitudinal forces) and left/right turns (lateral forces) of the virtual vehicle. We have designed a proof-of-concept system which is cost-effective and compact compare notably to vestibular platforms. A preliminary study, focused on a simple driving simulation in a straight line, showed that the presence of our haptic effects was globally preferred and improved the user experience. Taken together, our results suggest that this approach could be further tested and used in driving simulators in various entertainment and/or professional contexts.

\section{ACKNOWLEDGEMENTS}

The authors wish to thank Raoul Bernard, Mathilde Gilbert and Alexandre Jureidini from Telecom Sud-Paris and ENSIIE schools for their contribution.

\section{REFERENCES}

[1] S. G. T. Balter, M. H. Castelijns, R. J. Stokroos, and H. Kingma. Galvanic-induced Body Sway in Vestibular Schwannoma Patients: Evidence for Stimulation of the Central Vestibular System. Acta OtoLaryngologica, 124(9):1015-1021, nov 2004

[2] S. Bayarri, M. Fernandez, and M. Perez. Virtual reality for driving simulation. Communications of the ACM, 39(5):72-76, may 1996.

[3] A. Berthoz, B. Pavard, and L. Young. Perception of linear horizontal self-motion induced by peripheral vision (linearvection) basic characteristics and visual-vestibular interactions. Experimental Brain Research, 23(5):471-489, nov 1975.

[4] S. Birch. Mercedes-Benz' world class driving simulator complex enhances moose safety. 13 nov. 2010 (accessed 15 sept. 2016) http://articles.sae.org/9084/.

[5] J. E. Bos, J. van Erp, E. L. Groen, and H.-J. van Veen. Vestibulo-tactile interactions regarding motion perception and eye movements in yaw. Journal of Vestibular Research, 15(3):149-160, 2005.

[6] S. M. Cox, D. J. Cox, M. J. Kofler, M. A. Moncrief, R. J. Johnson, A. E. Lambert, S. A. Cain, and R. E. Reeve. Driving Simulator Performance in Novice Drivers with Autism Spectrum Disorder: The Role of Executive Functions and Basic Motor Skills. Journal of Autism and Developmental Disorders, 46(4):1379-1391, apr 2016.

[7] F. Danieau, J. Fleureau, A. Cabec, P. Kerbiriou, P. Guillotel, N. Mollet, M. Christie, and A. Lecuyer. Framework for enhancing video viewing experience with haptic effects of motion. In 2012 IEEE Haptics Symposium (HAPTICS), pages 541-546. IEEE, mar 2012.

[8] F. Danieau, J. Fleureau, P. Guillotel, N. Mollet, M. Christie, and L. Anatole. Toward Haptic Cinematography : Enhancing Movie Experience with Haptic Effects based on Cinematographic Camera Motions To cite this version :. IEEE MultiMedia, Institute of Electrical and Electronics Engineers (IEEE)., pages 11-21, 2014.
[9] F. Danieau, J. Fleureau, P. Guillotel, N. Mollet, A. Lécuyer, M. Christie, and A. Lécuyer. HapSeat: Producing Motion Sensation with Multiple Force-feedback Devices Embedded in a Seat. In Proceedings of the 18th ACM symposium on Virtual reality software and technology - VRST'12, page 69, New York, New York, USA, 2012. ACM Press.

[10] J. de Winter, P. van Leeuwen, and R. Happee. Advantages and Disadvantages of Driving Simulators : A Discussion. In A. Spink, F. Grieco, O. Krips, L. Loijens, L. Noldus, and P. Zimmerman, editors, Measuring Behavior, pages 47-50, Utrecht, The Netherlands, 2012.

[11] C. Dijksterhuis, D. de Waard, K. A. Brookhuis, B. L. J. M. Mulder, and R. de Jong. Classifying visuomotor workload in a driving simulator using subject specific spatial brain patterns. Frontiers in Neuroscience, 7, 2013

[12] I. Farkhatdinov, N. Ouarti, and V. Hayward. Vibrotactile inputs to the feet can modulate vection. In IEEE 2013 World Haptics Conference (WHC), pages 677-681. IEEE, apr 2013.

[13] D. Hallvig, A. Anund, C. Fors, G. Kecklund, J. G. Karlsson, M. Wahde, and T. Åkerstedt. Sleepy driving on the real road and in the simulatorA comparison. Accident Analysis \& Prevention, 50:44-50, 2013.

[14] B. Hassan, J. Berssenbrugge, I. Al Qaisi, and J. Stocklein. Reconfigurable driving simulator for testing and training of advanced driver assistance systems. In 2013 IEEE International Symposium on Assembly and Manufacturing (ISAM), pages 337-339. IEEE, jul 2013.

[15] Kirurobo. A c\# (.net) wrapper for sensable phantom device https://github.com/kirurobo/ManagedPhantom.

[16] W.-S. Lee, J.-H. Kim, and J.-H. Cho. A driving simulator as a virtual reality tool. In Proceedings. 1998 IEEE International Conference on Robotics and Automation, volume 1, pages 71-76. IEEE, 1998.

[17] T. Maeda, H. Ando, T. Amemiya, N. Nagaya, M. Sugimoto, and M. Inami. Shaking the world. In ACM SIGGRAPH 2005 Emerging technologies, page 17, Los Angeles, CA, USA, 2005. ACM Press.

[18] M. E. McCauley and T. J. Sharkey. Cybersickness: Perception of SelfMotion in Virtual Environments. Presence: Teleoperators and Virtual Environments, 1(3):311-318, jan 1992.

[19] N. Ouarti and V. Hayward. Specic vibrotactile signals in the feet enhance the experience of self-motion. In Theo Murphy Meeting on Active touch sensing, 2011.

[20] N. Ouarti, A. Lecuyer, and A. Berthoz. Haptic motion: Improving sensation of self-motion in virtual worlds with force feedback. In IEEE Haptics Symposium, HAPTICS, pages 167-174. IEEE, feb 2014.

[21] B. Pavard and A. Berthoz. Linear acceleration modifies the perceived velocity of a moving visual scene. Perception, 6(5):529-40, 1977.

[22] J. Rasmussen. Information Processing and Human-Machine Interaction: An Approach to Cognitive Engineering, 1986.

[23] G. Reymond and A. Kemeny. Motion Cueing in the Renault Driving Simulator. Vehicle System Dynamics, 34(4):249-259, oct 2000.

[24] B. Riecke, J. Schulte-Peikum, F. Caniard, and H. Bulthoff. Towards lean and elegant self-motion simulation in virtual reality. In IEEE Proceedings Virtual Reality, pages 131-138. IEEE, 2005.

[25] D. Salvucci. Predicting the effects of in-car interface use on driver performance: an integrated model approach. International Journal of Human-Computer Studies, 55(1):85-107, 2001.

[26] J. Sodnik, C. Dicke, S. Tomažič, and M. Billinghurst. A user study of auditory versus visual interfaces for use while driving. International Journal of Human-Computer Studies, 66(5):318-332, 2008.

[27] A. Valjamae, P. Larsson, D. Vastfjall, and M. Kleiner. Vibrotactile Enhancement of Auditory-Induced Self-Motion and Spatial Presence. Journal of the Audio Engineering Society, 54(10):954-963, 2006.

[28] F. Vienne, S. Caro, L. Désiré, J. Auberlet M, F. Rosey, and E. Dumont. Driving simulator: an innovative tool to test new road infrastructures. In TRA-Transport Research Arena, page 10p, 2014.

[29] B. G. Witmer and M. J. Singer. Measuring Presence in Virtual Environments: A Presence Questionnaire. Presence: Teleoperators and Virtual Environments, 7(3):225-240, jun 1998.

[30] E. Zeeb. Daimler's New Full-Scale, High-dynamic Driving Simulator A Technical Overview. In A. Kemeny, F. Merienne, and S. Espie, editors, Proceedings of the Driving simulation conference Europe 2010, pages 157-165, Paris, France, 2010. INRETS. 